\title{
Bilateral Energy Transfer for high fidelity haptic telemanipulation
}

\author{
Jordi Artigas * Carsten Preusche Gerd Hirzinger \\ Institute of Robotics and Mechatronics \\ DLR - German Aerospace Center \\ Germany, Munich
}

\author{
Gianni Borghesan Claudio Melchiorri \\ Università di Bologna \\ Bologna, Italy
}

\begin{abstract}
Among the methods to grant the stability of a telemanipulation system, the bilateral time domain passivity framework has the appealing characteristic to consider both the forces and velocities signal exchanged between the master and slave systems, and the power introduced or dissipated by the elements that compose the whole telemanipulation system. In previous works, [2,7], has been shown how the bilateral passivity controller (BiPC) can preserve stability when the communication channel that conveys data between master and slave is affected by delay. In this work the authors intend to further explore the possibilities offered by the Bilateral Energy Transfer concept as design guideline, and refine the control schemes already discussed in [7]. The underlying idea of Bilateral energy transfer is to achieve a transport of energy between the two sides of the real system as similar to an "ideal" (not delayed) system as it is allowed by the energy leaks introduced by the not ideal components, as the communication channel; in the meantime, in order to obtain easy-to-use system, the control system must preserve, in some extend, the force, velocity, and position correspondences between master and slave. In order to achieve this goal, a modified version of passivity controller is presented. The main characteristic of this controller is that its correction action aimed to dissipate energy, regarded as generated by energy leaks, is limited and deferred in time. Moreover, in this paper is introduced a drift compensator which role is to match the master and slave position; in order to maintaining the whole system passivity, the action of this controller is bounded to the amount of energy that has been dissipated in excess by the BiPC.
\end{abstract}

\section{INTRODUCTION}

The main goal of a telepresence system is to achieve transparency, meaning in its ideal form that the user is not able to distinguish remote presence to local presence. Besides vision and aural feedback the haptic channel is often presented to the user in order to render the tactile interaction upon the human operator which is taking place at the slave site. The haptic channel constitutes a key element in order to achieve realistic telepresence. Bilateral Control is the discipline which investigates the closed loop circuit created between the human operator and the remote / unreachable environment. Special control methods are hereby applied in order to stabilize an often very large loop whose communication delay (including the package loss phenomena), unavoidable non-linearities and the inclusion of a human operator in the control loop makes it specially challenging to tackle.

The pursuit of stability often compromises transparency once the system constraints are established. This trade-off is a common denominator in every single approach dealing with bilateral control. In this sense, one of the most accounted issues in haptic telemanipulation scenarios is the time delay that affects the communication channel. This fact often leads to the design of conservative control

*e-mail: jordi.artigas@dlr.de laws in order to achieve the unconditional system stability, which in turn often results in system transparency losses.

One of the most remarkable approaches in dealing with timedelayed telepresence is the passivity criteria. Passivity is a sufficient condition for stability and provides the nice feature that the system passivity is granted by the passivity of all its subsystems. Moreover, passivity of a system can be analyzed without an exact knowledge of its contents. It is therefore a useful tool which can be used as design rule in those systems where the communication channel becomes an active element due to the communication delay. A good example are the Scattering transformation [1] and its Wave Variables [8] formulation, which has become the classical approach in delayed teleoperation.

However most approaches that deal with delayed teleoperation end up using conservative techniques to detriment of the transparency and usability of the teleoperation system. In order to assure passivity and achieve a desired degree of transparency the bilateral control architectures often introduces elements which dissipate more energy than the strictly needed to compensate the energy introduced by the delayed communications. Wave variables -based methods, for instance, do have a non-lossy characteristic after applying the wave transformation; however, damping elements are then needed to minimize wave reflections and to achieve impedance matching [8] between master and slave.

This issue has been equally observed in bilateral passivity control -based methods [2, 3, 7, 9], based on Time Domain Passivity Control. Due to the impossibility of observing the real channel energy (unless using previous knowledge of the delay at both sides), forward and backward passivity controllers are forced to dissipate more energy than needed.

The methods presented in this paper use this rationale and compares the energy received by the slave with the energy that should be received; the difference between these energies, defined "wasted" in the sense that has been dissipated in excess, can be computed only in a delayed fashion. The wasted energy recovery controller injects then the energy which has been over-dissipated in order to strengthen the coupling of the master and slave position. This results in a more faithfull bilateral energy transfer [7] and thus in a transparency improvement.

Moreover, new kind of bilateral forward and backward passivity controllers, that well be referred with the prefix " $\Delta P$ ", are introduced; These $\Delta P$ controllers, in analogy with the PCDC, [7] and $\mathrm{PSH},[4]$, dissipates the energy that is estimated as generated; however, unlikely traditional the $\mathrm{PO} / \mathrm{PC},[5,6]$, the dissipation of extra energy is deferred in time, and, at each sample step, the average power $\Delta P$, that has to be dissipated in the given sample time, is computed in function of limit on the maximum distortion that is allowed on the modified signal. How much distortion can be introduced is modulated by a parameter, $\alpha$; in case of the parameter is set big enough, the $\Delta P$ - controllers behaves as a standard $\mathrm{PO} / \mathrm{PC}$, thus introducing spikes in particular critical conditions, while too small values compromise the ability of the controller to ensure stability. 


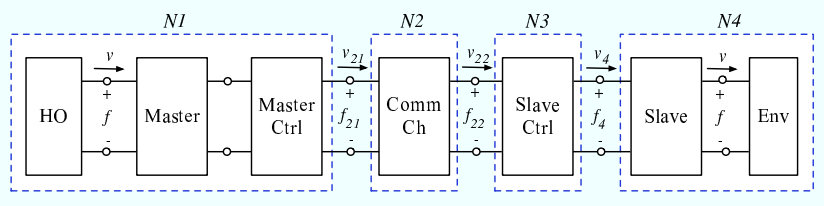

Figure 1: General network representation of a teleoperation system.

\section{SYSTEM DESCRIPTION}

Fig. 1 shows the network representation of a bilateral control system using the mechanical-electrical analogy. This scheme is general and resumes different possible causality choices, and includes the different elements normally involved in a teleoperation systems. Note that both the Human Operator (HO) and the Remote Environment (RE) are considered as part of the system, and are the only admissible sources of energy.

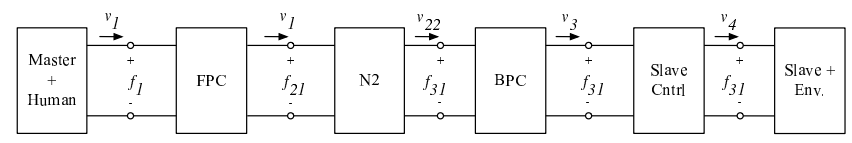

Figure 2: "Classic" Bilateral Energy Transfer scheme.

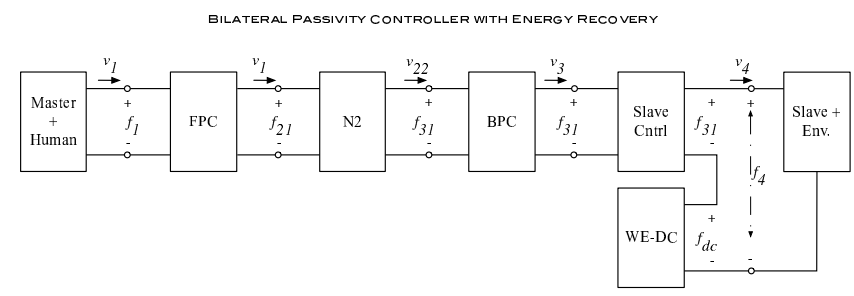

Figure 3: Network representation of the teleoperation system including the drift compensator.

As already pointed out, this paper is an incremental work that starts from the scheme depicted in Fig. 2, where it is possible to observe the insertion of the Forward and Bilateral Passivity Controllers, whose role is to passivate the channel. it is possible to see that, as design choice, the master side controller has not been included; the system causality is set so that the master and slave output are velocities, while the slave controller produces forces. The FPC output is the force $f_{1}$ while the BPC output is $v_{3}$, and the other signals $\left(v_{1}\right.$ and $\left.f_{31}\right)$ are untouched by the passivity controllers, as can be seen in Fig. 2.

The system that will be studied in the remainder of this paper is represented in Fig. 3; the main differences are the new specification of the BPC and FPC, and the insertion a novel element of where the different role of the various elements is briefly introduced:

- Master + Human: Contains the Human Operator , the master device, and the analog/digital conversion plus the velocity estimation from the robot position (AD/DA - VEL. EST.), and it is usually the source of the system. (1-port).

- N2: Is the Communication Channel. (2-port).

- Slave Cntrl: The slave controller is a virtual coupling. (2port).

- $W E-D C$ : Is the Drift Compensator based the on wasted energy (see Sec. 5). (1-port).
- Slave + Env:: Includes the remote environment (RE), the slave Robot and its AD/DA - VEL. EST., and it is usually a passive system. (1-port).

- FPC and BPC:Are $\triangle P$ Forward and Backward Passivity Controllers (see Sec. 4).

In this work also the PCDC presented in [7] is employed and inserted both in the master and slave subsystems, but will be omitted in the rest of the paper for sake of clarity. Its role is to correct the energy generated in the robot velocity estimation from sampled data, in order to ensure the passivity of such operation.

Following the criteria of the bilateral energy transfer, the virtual coupling is tuned in order to grant stability and desired performance in the ideal case, where all the sources of energy leaks are not considered; in particular, no delay is present in the control loop and the system is considered in the continuous time domain [2], so that the effects of time discretization is ignored; in this case, the effects of the various controllers (FPC, BPC and WE-DC) are not taken into account. Instead, the force computed by the virtual coupling is directly applied to both, slave and master.

In Fig. 3 it is possible to see the power correlated signals, forces and velocities. The product of these signals are powers, so from their integration it is possible to compute the net energy flowing trough any given port; the following convention with regard to the sign of energy is used; a 1-port element is passive if:

$$
E(t)=\int_{0}^{t} f(\tau) v(\tau) d \tau+E(0) \geq 0,
$$

where $E(t)$ is the energy of the network, $v$ and $f$ are the port variables denoting velocity entering the block, and force respectively, and $E(0)$ is the energy initially stored in the network at $t=0$. So in the case the energy source of the system is N1 (the master robot and human operator), its energy, $E_{N 1}(t)$, traces a negative slope indicating the active behavior.

\section{Bilateral Energy Transfer}

This section introduces the main idea behind this work: the energy transfer from the master side to the slave side of the teleoperation system. The ideal system behaves as two masses, the master and slave robots, connected by the virtual coupling, a spring-damper system. The introduction of non-idealities brings the system to behave differently, in particular from the point of view of energy flows, where delay, position discretization introduces energy in the system possibly bringing it to the verge of instability and over.

It has been shown in [7] that by using the Passive Discrete Connector in conjunction with Forward and Backward Passivity Controllers the commanded energy from the human operator can be transfered to the slave system. However, the strategy used in order to keep the channel passive and due to time the delay uncertainty property of the bilateral passivity controller (i.e. the amount of time delay is not a parameter of the controller and can be thus unknown and variable), the produced energy in the channel is sometimes over dissipated. This lossy nature of the system ensures stability but has an effect upon performance which is mainly translated in a position drift between master and slave position signals.

Fig. *** shows a comparison between the energy produced by $\mathrm{N} 1$ (i.e. the master), and what after the BPC comes. This energy loss is responsible for transparency degradation. Taking a sharp look at the Backward Passivity Controller presented in [7], it is clear that if more energy than the needed one is dissipated this will outcome in a position drift, since the causality conserves forces and outputs modified velocity.

The methods proposed in this article tackle this issue and, into some extend, turn the system to behave as expected, that is to transfer the kinetic energy from the master "mass" to the virtual damper and the slave "mass". 


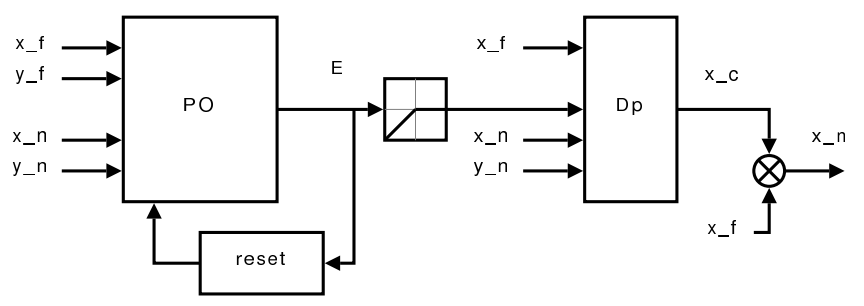

Figure 4: General structure of the $\Delta P$ PC.

\section{Description of the $\Delta P$ Forward AND Bilateral PASSIVITY CONTROLlER}

The $\Delta P$ PC is described as the cascade of functional blocks, as shown P Fig. 4, for sake of clarity. In this section the generic power correlated signals $x$ and $y$ will be used instead of force and velocity, that are specified by the system causality. Since The $\Delta P$ PC is designed to passivate a two-ports network and, therefore, needs two couples of power correlated signals as input: one signal couple, $x_{n}$ and $y_{n}$, is not divided by delay blocks (in the case at hand by the transmission channel between the local and remote systems) by $\Delta P$ PC, and therefore defined "near". The other signal couple, $x_{f}$ and $y_{f}$ can be known by the only $\Delta P$ PC with delay and therefore defined as "far". The $\Delta P$ PC modifies one of the "near" signal, the choice of which signal is the one corrected depends upon the system causality: that signal is the output signal of "near" port of the controlled two-ports system; in the case of the BPC the near signals are $f_{1}$ and $v_{1}$, and the output is $f_{1}$ while the far signals are $v_{22}$ and $f_{3} 1$, that can be known only passing through the channel and therefore are affected by delay. The $\Delta P \mathrm{PC}$ is divided in three functional sub-blocks:

- The Passivity Observer, that estimates the energy of the two port system that must be passivated,

- The Reset, that produces the reset signal for the PO,

- The $\Delta P$ block, that computes the average power $\Delta P$ that must be dissipated in the next sample time, and also computes the correction term to be applied on one of the "near" signal.

The $\Delta P$ PC is designed to work as discrete time system, with a sample time of $T_{S}$

In the following the description of the blocks different roles is given.

Passivity Observer: It estimates the energy inside the two port system under analysis; the energy cannot be computed exactly, since the "far" signals are accessible only after a delay, instead a rough approximation is considered not taking into account the delay.

$$
E(n)=\sum_{k=-\infty}^{n} T_{s}\left(x_{n}(k) y_{n}(k)-x_{f}(k) y_{f}(k)\right)
$$

The value of the integrator is reset each time the reset signal is produced by the Reset block.

Reset: it produces the reset signal each time the value of $E$ is positive AND its derivative is negative. In this way the accumulation of passive energy is limited, thus allowing a faster passivity recovery. $\Delta P:$ it computes the power that is dissipated in a sample time and consequently the correction $x_{c}$ to be applied to the passivated system output $x_{n}=x_{f}+x_{c}$. Note that the energy $E$ used as input is saturated, in order to force a dissipative behavior. The algorithm employed to design this block is inspired to the PCDC and PSH presented in $[4,7]$; in particular it uses the same idea to limit the distortion of the output signal $x_{n}$ respect to the input signal $x_{f}$ enforcing the following inequality:

$$
\left|x_{n}(k)-x_{n}(k-1)\right| \leq \alpha\left|x_{f}(k)-x_{f}(k-1)\right|
$$

where $\alpha>0$ is a multiplicative coefficient that can enforce or relax the condition imposed by (3); in the PCDC this value was fixed as one. The output of this block is a correction $x_{c}$, defined as:

$$
x_{c}(k)=\Delta P(k) / y_{f}(k)
$$

Where $\Delta P(k)$ is the energy that is dissipated in a sample time $T_{s}$ From (3) and (4), the following is obtained:

$$
\left|x_{f}(k)+\frac{\Delta P(k)}{y_{n}(k)}-x_{n}(k-1)\right| \leq \alpha\left|x_{f}(k)-x_{f}(k-1)\right|
$$

After applying the inverse triangular equation the first upper bound $^{1}$ of $\Delta P$ is obtained:

$$
\left.\left|\frac{\Delta P}{y_{n} k}\right| \leq \alpha\left|x_{f}(k)-x_{f}(k-1)\right|+\mid x_{f}(k)-x_{(} k-1\right) \mid
$$

The value $|\Delta P|$ is also limited by the amount of energy $E(k)$ observed.

$$
T_{s} \Delta P(k) \leq|E(k+1)|
$$

Differently from the PCDC, the sign of the energy is always negative, so also the sign of $\Delta P(k)$ is chosen negative, while its absolute value is the maximum that satisfies both (6) and (7).

At this point, the output is computed with (8) instead of (4), in order to avoid the zero division:

$$
x_{c}= \begin{cases}\Delta P / y_{f} & \left|y_{f}\right|>y_{t r} \\ 0 & \left|y_{f}\right| \leq y_{t r}\end{cases}
$$

where $y_{t r}>0$ is the threshold value under whose value no correction is applied to $x_{f}$.

\section{Drift Compensator: Wasted energy Recovery}

The use of Bilateral Passivity Controller has a dichotomy between what is considered a great advantage, and a drawback: the unneeded information of the time delay value on one side; and the lossy nature of the delay compensation through the bilateral passivity controller on the other. The second one is actually a consequence of the first one, since using previous knowledge of the time delay allows exact computation of energy produced in the channel. However it is important to keep independence from the delay value, since in real space links, [12], or Internet scenarios ( [11], Chap. 14), it is hard, if not impossible, to predict it.

The strategy here proposed is based on an observer of both commanded energy by master haptic device and received energy at the slave site. As afore mentioned, the second one is often a portion of the first one due to the over dissipative nature of the BPC and FPC.

The difference between commanded and received energy is the wasted energy. This energy loss produces the drift in position, first observed in [7], since the BPC conserves force and dissipates upon velocity, i.e. it slows down slave motions. However, if the wasted energy is computable, this one can be used as a boundary up to which extra energy can be inserted in order to compensate the drift.

For the sake of simplicity, the analysis is limited to a delay in the forward path and with the FPC deactivated. In this case the received energy at the right port of the BPC becomes:

\footnotetext{
${ }^{1}$ Since only an upper bound is sought, the lower bound is discarded
} 


$$
\begin{aligned}
E_{r x}(n) & =E_{N 1}(n)+E_{N 2}(n)+E_{B P C}(n)= \\
& =\Delta T_{s}\left[\sum_{k=1}^{n}-f_{1}(k) v_{1}(k)+\right. \\
& +\sum_{k=1}^{n}-f_{31}(k) v_{1}(k-T)-f_{31}(k-T) v_{1}(k)+ \\
& \left.+\sum_{k=1}^{n} f_{31}(k) v_{22}(k)-f_{31}(k-T) v_{1}(t-T)\right],
\end{aligned}
$$

which after equating for $f_{31}(k)$ and $v_{1}(t-T)$ it becomes:

$$
E_{r x}(n)=\Delta T_{S}\left[\sum_{k=1}^{n}-f_{31}(k-T) v_{1}(k-T)\right] .
$$

If energy was ideally transfered then

$$
E_{r x}(n)=E_{N 1}(n)
$$

However, it can be seen how actually the ideal value differs from the real one, since:

$$
\begin{aligned}
E_{N 1}(n)-E_{r x}(n) & =\Delta T_{s}\left[\sum_{k=1}^{n} f_{31}(k-T) v_{1}(k)+\right. \\
& \left.+f_{31}(k-T) v_{1}(k-T)\right] \\
& =\Delta T_{s}\left[\sum_{k=1}^{n} f_{31}(k-T)\left[-v_{1}(k)+v_{1}(n-T)\right] .\right.
\end{aligned}
$$

This corresponds to the energy dissipated in excess by the BiPC, i.e the wasted energy. A new control element can be therefore introduced. This element injects energy to the slave system in order to correct the drift without overcoming the wasted energy (otherwise the overall system would not be passive).

In particular, a virtual spring placed between the not yet modified velocity signal coming straight from communication channel, and the current velocity right next to the BPC, can be used to generate the force needed to correct the position drift. Fig. 3 and Fig. 5 show the operation of the proposed scheme.

The drift compensator is thus defined as a PI controller:

$$
f_{d c}^{*}(t)=K_{i} \int_{0}^{t}\left[v_{22}(\tau)-v_{3}(\tau)\right] d \tau+K_{p}\left(v_{22}(t)-v_{3}(t)\right),
$$

where $K_{p}$ and $K_{i}$ are proportional and integral gains. This controller has a different nature respect to the slave controller, because the drift compensator employs velocities that are taken in different parts of the system and therefore the drift compensator can be considered as a modulated force generator; this fact is due to that $v_{22}$ and $f_{4}$ are not power correlated and do not individuate a power port, as can be seen in Fig. 3.

Being a force generator this system can be both active and passive; as hinted before, in order to keep the whole teleoperation system passive, the energy must be negative (passive) or bounded by energy dissipated in some other part of the system. For this reason $K_{p}$ and $K_{i}$ are tuned empirically so that (11) approximately fulfills, however if it is not happen, by means of a switch controlled by the computed wasted energy, the injected energy will never overcome the permissible one. This is:

$$
f_{d c}(t)=\left\{\begin{array}{lr}
f_{d c}^{*}(t) & E_{d c}(t)>E_{N 1}(t)-E_{r x}(t) \\
0 & \text { else }
\end{array}\right.
$$

Enforcing the condition expressed by (14) the passivity of the whole system is preserved, even if an active block is introduced; this a somewhat similar method has been employed in [10], where wave variables based telemanipulation system is considered.

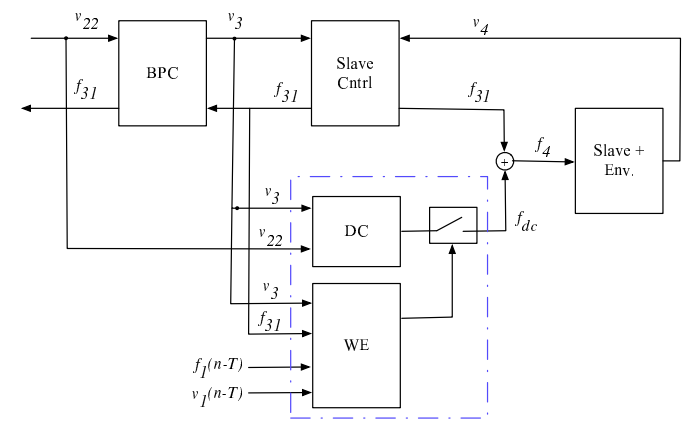

Figure 5: Operation of the Drift Compensator.

\section{Simulations}

Master and slave robots are simulated (Matlab Simulink) as mass-damper systems with mass $M_{r}=1 \mathrm{Kg}$ and viscous friction of $B_{r}=0.3 \mathrm{Ns} / \mathrm{m}$. The $\mathrm{HO}$ is simulated in a closed-loop system that generates the force to the master robot in order to follow a sinusoidal reference position with frequency of $0.25 \mathrm{~Hz}$ and amplitude of $0.25 \mathrm{~m}$. The control part of the system (PCDCs, FPC, $\mathrm{BPC}$ and $\mathrm{N} 3$ ) runs at a sampling rate of $T_{s}=1 \mathrm{~ms}$.

Fig. 6 shows the system under a round trip of delay of 500 ms using the redefined BPC and FPC and the drift compesnator disables. There is a gain in performance in compared to previous implementations of the BiPC [7]. The tranparency increment can be clearly seen in the force signal, which become less noisy due to the modulated dissipation along the the time axis. Fig 7 shows the energies of the system. Here the over-dissipatvie nature of the BiPC can comparing both, $E_{N 1}$ and $E_{N 4}$. Futher, the wasted energy can be seen in Fig. 8, as the difference between $E_{r x}$ and $E_{N 1}$.

Fig. 9 shows the system under a round trip of delay of $500 \mathrm{~ms}$, again using the redefined BPC and FPC and the drift compesnator based on energy recovery activated. The position tracking becomes closer to what is expected, resulting in finer motions and relax forces.The operation of both, forward and backward passivity controllers can be seen in Fig. 11. Further the system energies, plotted in Fig. 10, show a more faithfull energy transfer takeing place between master and slave. This benefit is equally corroborated looking at the energy recovery in Fig. 12.

Fig. 13 shows the system under a round trip of delay of $500 \mathrm{~ms}$, again using the redefined BPC and FPC and the drift compensator based on energy recovery activated and in a contact situation against a virtual wall with stiffness $300 \mathrm{~N} / \mathrm{m}^{2}$.

\section{Conclusion}

In this work, the authors further exploit the potentiality of the Bilateral Time Passivity approach, in order to obtain a more faithful energy transfer in a delayed teleoperation scenario respect to the previous results. To do so, a force velocity architecture has been considered, and two controllers, that strongly relies on the energy computation or estimation in the networks acts to grant the passivity of the system, achieving also fidelity on the movements of master and slave robots. The above controllers are a modified version of the Bilateral Passivity Controller, defined as $\Delta P$ controller, that takes care of passivate the delayed channel; its novel characteristic 
consist in modulate the dissipation along a length of time so that brusque control actions are avoided. The second controller instead is a PI controller, that employs the energy that has been dissipated in access due to the necessary over conservatism of the passivity controllers, in order to correct the position drift. This combination of controllers given satisfactory results in simulation. Still, this works has possible development, as an extensive experimental test, and the design of rules to automatically and dynamically change of important parameters, such as the $\alpha$ that limit the distortion of the corrected signal but also can cause instability if taken not sufficiently high; also, a method to tune the gains of the drift compensator must be sought.

\section{REFERENCES}

[1] R. J. Anderson and M. W. Spong. Bilateral control of teleoperators with time delay. IEEE Transactions on Automatic Control, 34(5):494501, May 1989.

[2] J. Artigas, C. Preusche, and G. Hirzinger. Time domain passivity for delayed haptic telepresence with energy reference. In IEEE/RSJ International Conference on Intelligent Robots and Systems., San Diego, CA, USA, 2007.

[3] J. Artigas, J. Vilanova, C. Preusche, and G. Hirzinger. Time domain passivity - based telepresence with time delay. In IEEE International Conference on Intelligent Robots and Systems, 2006.

[4] G. Borghesan, A. Macchelli, and C. Melchiorri. Simulation issues in haptics. In Proc. IEEE ICRA '07, 2007.

[5] B. Hannaford and J.-H. Ryu. Time domain passivity control of haptic interfaces. In Proc. IEEE ICRA '01, pages 1863-1869, 2001.

[6] B. Hannaford, J.-H. Ryu, and Y. S. Kim. Sampled- and continuoustime passivity and stability of virtual environments. IEEE Trans. on Robotics, 20,4:772- 776, 2004.

[7] C. P. Jordi Artigas, G. Hirzinger, G. Borghesan, and C. Melchiorri. Bilateral energy transfer in delayed teleoperation on the time domain. In Proc. IEEE ICRA '08, 2008.

[8] G. Niemeyer. Using Wave Variables in Time Delayed force Reflecting Teleoperation. PhD thesis, Massachussetts Institute of Technology, Sept. 1996

[9] J.-H. Ryu, B. Hannaford, C. Preusche, and G. Hirzinger. Time Domain Passivity Control with Reference Energy Behavior. In Proceedings of the 2003 IEEE/RSJ Intl. Conference on Intelligent Robots and Systems, Las Vegas, Nevada, USA, October 2003.

[10] C. Secchi, S. Stramigioli, and C. Fantuzzi. Position drift compensation in port-hamiltonian based telemanipulation. In IEEE/RSJ International Conference on Intelligent Robots and Systems., San Diego, CA, USA, 2006.

[11] C. Secchi, S. Stramigioli, and C. Fantuzzi. Control of interactive robotic interfaces A port-Hamiltonian approach, volume 29 of Springer Tracks in advanced robotics. Springer Verlag, New York, 2007.

[12] E. Stoll, J. Artigas, J. Letschnik, C. Preusche, U. Walter, and G. Hirzinger. Ground verification of the feasibility of telepresent onorbit servicing. Journal of Field Robotics, 2008.
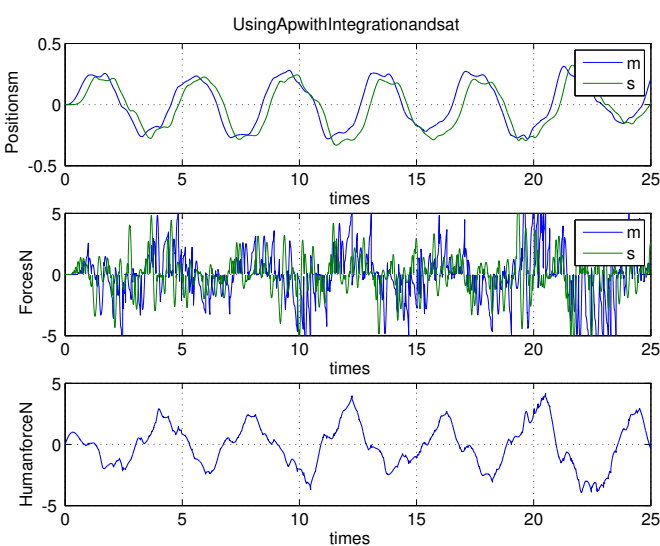

Figure 6: Position and forces. Delay $=500 \mathrm{~ms}$. BiPC activated. Drift Comp. disabled
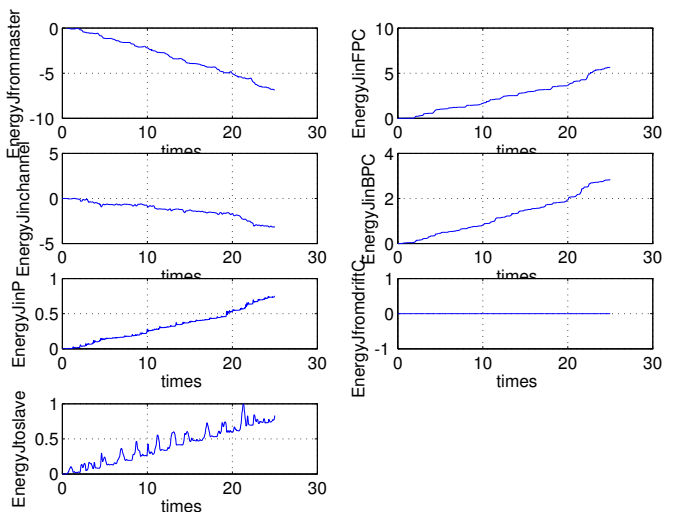

Figure 7: Network energies. Delay $=500 \mathrm{~ms}$. BiPC activated. Drift Comp. disabled
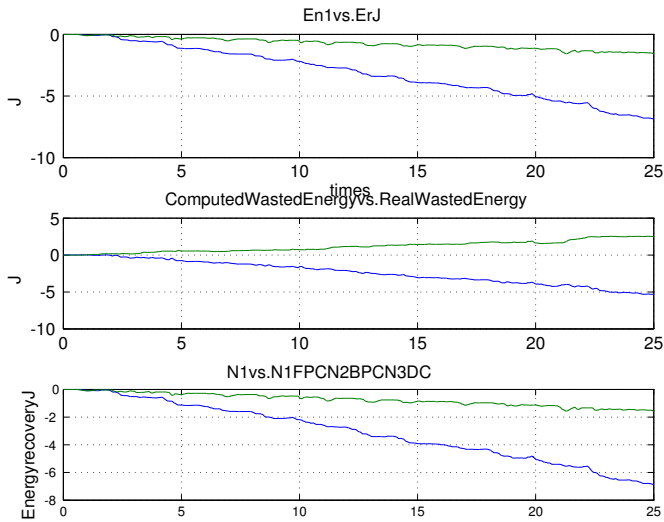

Figure 8: Wasted Energy. Delay $=500 \mathrm{~ms}$. BiPC activated. Drift Comp. disabled 

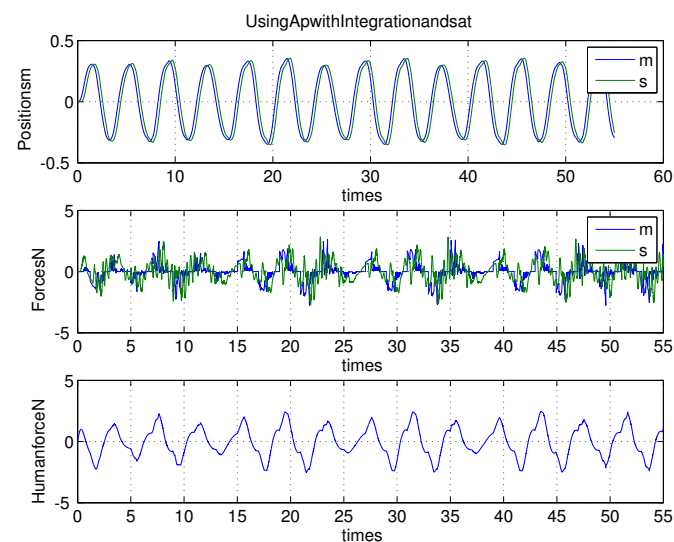

Figure 9: Position and forces. Delay $=500 \mathrm{~ms}$. BiPC activated. Drift Comp. activated
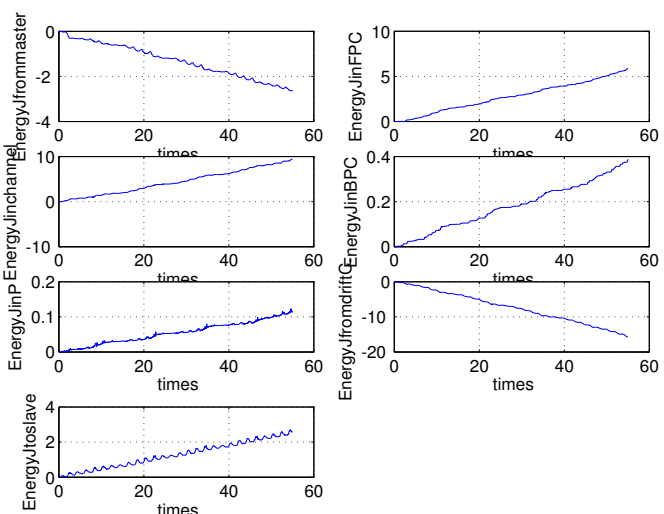

Figure 10: Network energies. Delay $=500 \mathrm{~ms}$. BiPC activated. Drift Comp. activated
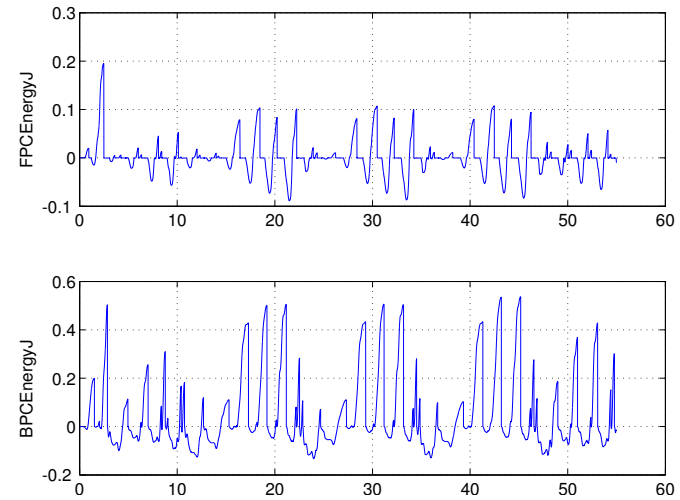

Figure 11: FPC and BPC energies. Delay $=500 \mathrm{~ms}$. BiPC activated. Drift Comp. activated
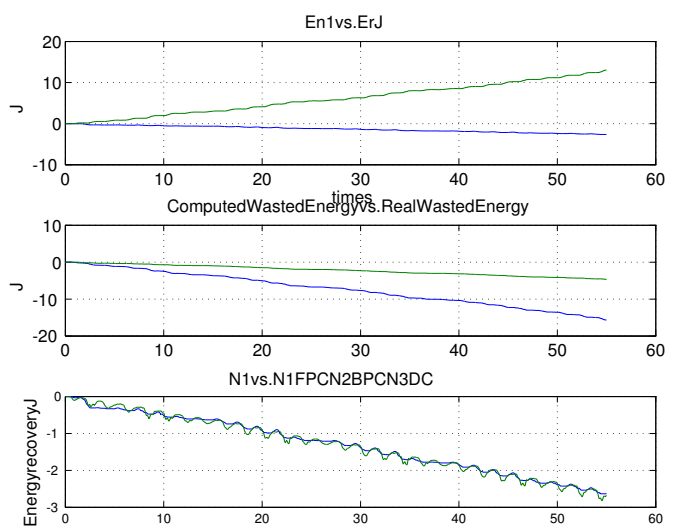

Figure 12: Wasted Energy. Delay $=500 \mathrm{~ms}$. BiPC activated. Drift Comp. activated
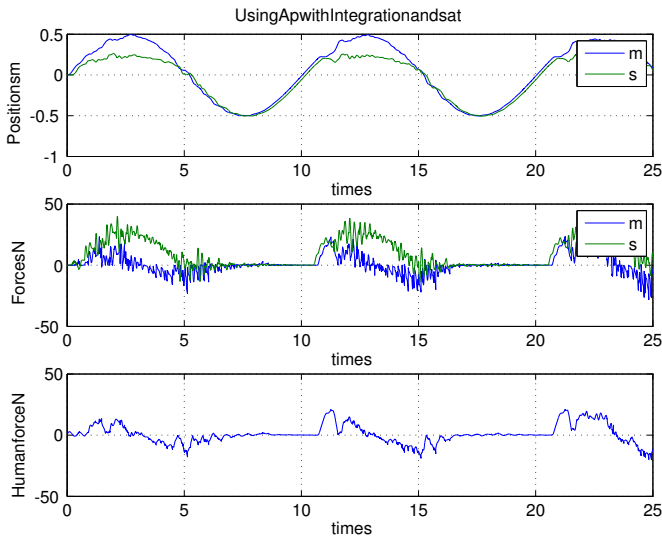

Figure 13: Position and forces in contact situation. Delay $=500 \mathrm{~ms}$. BiPC activated. Drift Comp. activated 